\title{
Eccema dishidrótico palmoplantar asociado a secukinumab
}

\section{Palmoplantar dyshidrotic eczema related to secukinumab}

Fiorella Bertani ${ }^{1}$, Laura Weintraub ${ }^{2}$, María Laura García Bazarra ${ }^{2}$, Graciela Sánchez $^{3}$ y Gladys Merola ${ }^{4}$

\section{RESUMEN}

El uso de agentes biológicos para el tratamiento de la psoriasis permite desarrollar una estrategia terapéutica a largo plazo, pero, como resultado de un posible desbalance en los perfiles de respuesta inmune, introducen nuevos efectos colaterales. Las reacciones adversas cutáneas relacionadas al uso de secukinumab son infrecuentes, las más comunes son la urticaria y las infecciones. Se presenta el caso de un paciente con psoriasis que mostró una dermatitis vesiculosa palmoplantar luego del inicio del tratamiento con secukinumab.

Palabras clave: secukinumab, eccema dishidrótico palmoplantar, dishidrosis palmoplantar.

Dermatol. Argent. 2020, 26 (4): 176-178

\section{ABSTRACT}

The use of biological agents for the treatment of psoriasis makes it possible to develop a long-term therapeutic strategy, but as a result of a possible imbalance in the immune response profiles it introduces new adverse effects. Cutaneous adverse reactions related to the use of secukinumab are rare, the most common being urticaria and infections. We present the case of a patient with psoriasis who manifested palmoplantar vesicular dermatitis after starting treatment with secukinumab.

Key words: secukinumab, palmoplantar dyshidrotic eczema, palmoplantar dyshidrosis.

Dermatol. Argent. 2020, 26 (4): 176-178
${ }^{1}$ Médica Especialista en Dermatología

${ }^{2}$ Médica de Planta. Especialista en Dermatología

${ }^{3}$ Médica Anatomopatóloga

${ }^{4}$ Jefa del Servicio de Dermatología

Hospital General de Agudos Dr. Teodoro Álvarez, Ciudad

Autónoma de Buenos Aires, Argentina
Contacto del autor: Fiorella Bertani

E-mail: fio.bertani@gmail.com

Fecha de trabajo recibido: $27 / 10 / 2020$

Fecha de trabajo aceptado: 14/12/2020

Conflicto de interés: las autoras declaran que no existe conflicto de interés.

\section{CASO CLÍNICO}

Paciente de sexo masculino de 72 años, oriundo de Buenos Aires y de ocupación taxista, en seguimiento por nuestro Servicio por psoriasis vulgar grave de 27 años de evolución. Recibió tratamiento con fototerapia, metrotexato y acitretín, de manera consecutiva, con fallo secundario en todos los casos. Como antecedente personal presentaba hipertensión arterial tratada con enalapril, sin otras comorbilidades. En 2017, concomitante a la falla terapéutica al acitretín, se diagnosticó espondiloartropatía psoriásica. Por ese motivo se decidió, junto con el Servicio de Reumatología, suspender el acitretín e iniciar tratamiento con secukinumab, previa evaluación y exclusión de contra- indicaciones. Al inicio del tratamiento el PASI fue de 16 , con reducción a PASI 0 a los 3 meses.

$\mathrm{Al}$ año del inicio del tratamiento con secukinumab, el paciente presentó una dermatosis de aparición aguda y localización palmoplantar caracterizada por eritema, vesículas, descamación y costras (Fotos 1 y 2). Se encontraba en buen estado general, sin lesiones de psoriasis ni síntomas artropáticos. Negó el uso de agentes tópicos en las palmas y plantas o cambios en referencia a su ocupación. El cuadro se interpretó como un eccema dishidrótico y se indicó tratamiento con clobetasol tópico oclusivo y emolientes. Después de un mes de tratamiento el paciente no mostró mejoría significativa. Se solicitó un laboratorio que resul- 
tó normal y la realización de una biopsia cutánea. Los diagnósticos presuntivos fueron psoriasis paradojal versus efecto adverso asociado al secukinumab. El informe anatomopatológico describió: epidermis con presencia de vesículas y espongiosis, leve exocitosis linfocitaria y dermis con marcada cantidad de eosinófilos, compatible con eccema dishidrótico (Fotos 3 y 4). En el intervalo de tiempo entre la toma de la muestra cutánea y el resultado, el paciente falleció como consecuencia de un accidente cerebrovascular, por lo tanto, no se pudo suspender el secukinumab ni revaluar el cuadro.

El paciente no tenía historia previa de dishidrosis, no asoció nueva medicación ni cambios de hábitos y se descartó una infección desencadenante. Por otra parte, la biopsia mostraba una llamativa cantidad de eosinófilos en la dermis, asociada a la falta de respuesta a corticosteroides tópicos. Se interpretó como una reacción secundaria al tratamiento con secukinumab.

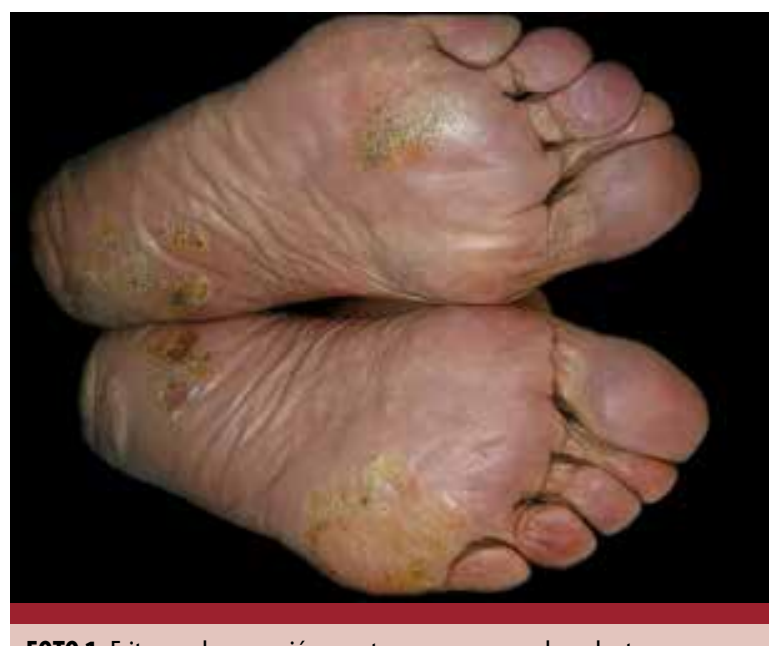

FOTO 1: Eritema, descamación y costras serosas en ambas plantas.

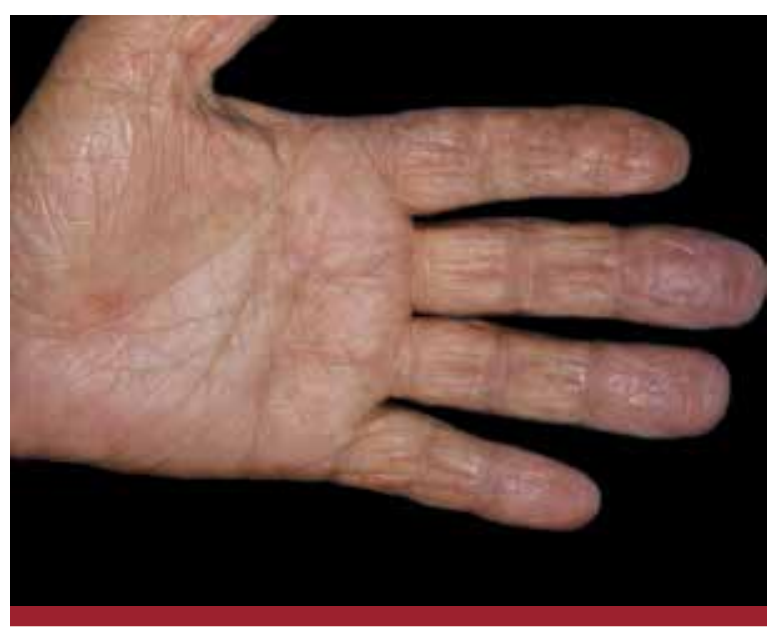

FOTO 2: Pápulas eritematosas en la región superior de la palma de la mano. Erosiones y leve descamación en eminencias tenar e hipotenar. Costras serosas en tercera falange del dedo índice.

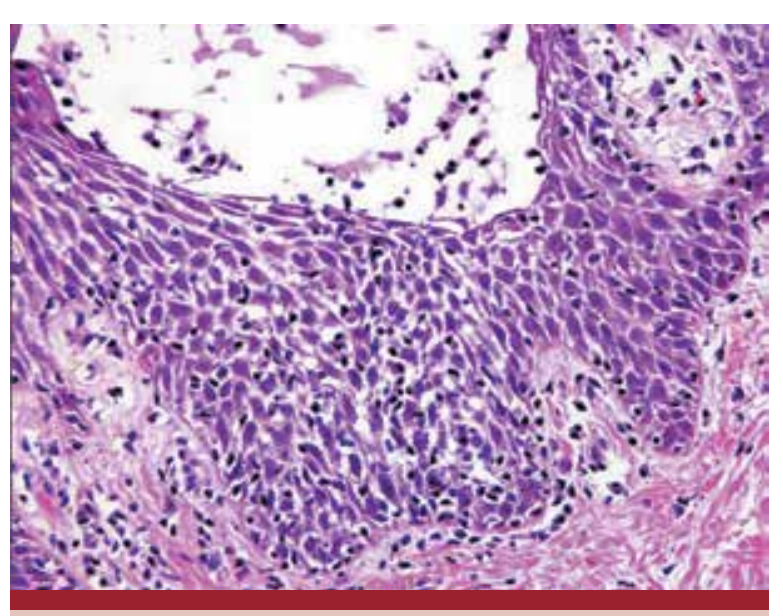

F0T0 3: Epidermis con presencia de vesículas y espongiosis, leve exocitosis linfocitaria (HyE, 250X).

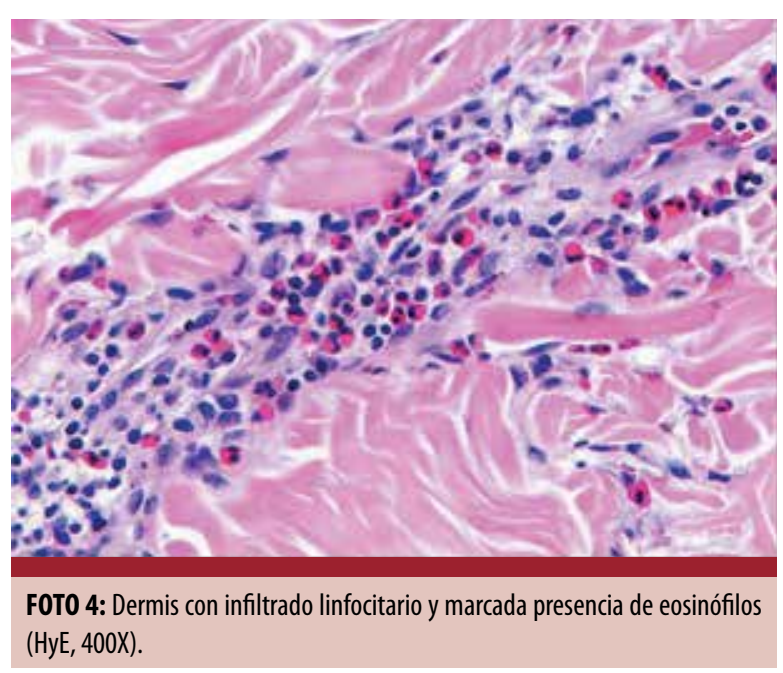

\section{COMENTARIOS}

La psoriasis se manifiesta en la piel con lesiones eritematoescamosas debidas a la proliferación exacerbada de queratinocitos, proceso mediado por citocinas proinflamatorias, como la IL-17 A. En la piel de los pacientes con psoriasis, esta citoquina conduce al reclutamiento de células inflamatorias, proliferación epidérmica y disrupción de la barrera cutánea ${ }^{1,2}$.

El secukinumab es un fármaco eficaz en el tratamiento de la psoriasis, ya que inhibe la vía de la IL-17 y logra una reducción de PASI 100 en el 36\% de los pacientes tratados en un período de 3 meses, como sucedió en nuestro caso ${ }^{2,3}$.

El eccema dishidrótico es una dermatosis inflamatoria crónica con múltiples causas (dermatitis atópica preexistente, dermatitis de contacto, estrés y desencadenantes infecciosos). Su patogénesis no se encuentra completamente dilucidada; sobre la base de los hallazgos cutáneos se sugiere que sería un proceso mediado por el perfil de acción Th2 $2^{4}$.

Es posible que la inhibición que produce el secukinumab a la IL-17, al bloquear la vía Th17, genere un desba- 
lance entre los perfiles de respuesta inmune y propicie la aparición de otras dermatosis; en este caso, por aumento de la respuesta $T h 2$ de un eccema dishidrótico ${ }^{4}$.

Los efectos adversos observados durante el tratamiento con secukinumab en estudios en fase 3 fueron: nasofaringitis, diarrea, infecciones de la vía aérea superior, rinitis, herpes oral, faringitis, urticaria y rinorrea. Otros efectos adversos cutáneos presentados en menor proporción incluían tiña pedis, candidiasis oral e impétigo ${ }^{5}$. Existen escasas publicaciones de otras manifestaciones en la piel ${ }^{6,7}$. En 2019, Blackcloud et ál. publicaron la primera asociación de secukinumab y dermatitis palmoplantar ampollar ${ }^{8}$.

Utilizamos la Escala de Naranjo para evaluar la asociación entre el fármaco y la reacción adversa9. El valor obtenido en este paciente fue 4, ya que existen notifica-

\section{BIBLIOGRAFÍA}

1. Li J, Chen X, Liu Z, Yue Q, et ál. Expression of Th17 cytokines in skin lesions of patients with psoriasis. J Huazhong Univ Sci Technolog Med Sci 2007;27:330-332.

2. Lavieri A, Acevedo A, Baccarini E, Bourren P, et ál. Consenso nacional de psoriasis. Sociedad Argentina de Dermatología. 2020 [en línea]. Disponible en https://sad.org.ar/wp-content/ uploads/2020/09/CONSENSO-NACIONAL-DE-PSORIASIS.ACTUALIZACION-2020.-.pdf (Consulta: setiembre 2020).

3. Reich K, Warren RB, Coates LC, Di Comite G. Long-term efficacy and safety of secukinumab in the treatment of the multiple manifestations of psoriatic disease. J Eur Acad Dermatol Venereol 2020;34:1161-1173.

4. Bose R, Beecker J. Dyshidrotic eczema in two patients on secukinumab for plaque psoriasis: A case report. SAGE Open Med Case Rep 2020;8:1-4. ciones de esta reacción en la literatura médica. Presentó la reacción posteriormente a la administración del fármaco y esta fue confirmada por biopsia cutánea ${ }^{4,8}$. Sin embargo, es importante tener en cuenta que algunos de los puntos que evalúa esa escala no pudieron determinarse, ya que el paciente no había recibido previamente un fármaco con acción anti-IL-17 y no pudo medirse la concentración sérica del secukinumab. Adicionalmente, fallece a causa de un accidente cerebrovascular, por lo que no se pudo suspender el fármaco ni evaluar la respuesta.

La aprobación del secukinumab para el tratamiento de la psoriasis vulgar en placas moderada a grave es reciente, por lo tanto, debemos estar alertas ante este posible efecto adverso. Este es el primer caso de dishidrosis palmoplantar asociado al uso de secukinumab en la Argentina.

5. Pariser D, Frankel E, Schlessinger J, Poulin Y, et ál. Efficacy of Secukinumab in the Treatment of Moderate to Severe Plaque Psoriasis in the North American Subgroup of Patients: Pooled Analysis of Four Phase 3 Studies. Dermatol Ther (Heidelb) 2018;8:17-32.

6. Sladden MJ, Sladden CS, Gulliver WPF. Secukinumab-Induced Psoriasiform Eruption. JAMA Dermatol 2017; 153:1194-1195.

7. Ho PH, Tsai TF. Development of bullous pemphigoid during secukinumab treatment for psoriasis. J Dermatol 2017;44:e220-e221.

8. Blackcloud P, Dupuy E, Kang Y, Smart C, et ál. Bullous acral eruption related to secukinumab. Dermatol Online J 2019; 25:1-4.

9. Naranjo CA, Busto U, Sellers EM, Sandor $P$, et ál. A method for estimating the probability of adverse drug reactions. Clin Pharmacol Ther 1981;30:239-245.

\section{PERLAS}

Federico Pastore

Médico Dermatólogo y Legista. Médico de Planta de Dermatología, Hospital Dr. Enrique Tornú. Jefe del Servicio de Dermatología, Instituto César Milstein

EFICACIA Y SEGURIDAD DEL MINOXIDIL ORAL A BAJAS DOSIS EN HOMBRES CON ALOPECIA ANDROGÉNICA

El minoxidil oral se utiliza solo o combinado con otras terapéuticas para el tratamiento de la alopecia androgénica en hombres y mujeres. La dosis diaria varía entre 0,25 mg y 2,5-5 mg.

En este estudio se utilizó una dosis de 1,25 mg/día en monoterapia durante 24 semanas.

Se incluyeron 32 pacientes de 18 a 45 años, de los cuales $18(56,2 \%)$ nunca habían recibido tratamiento, mientras que en $14(43,8 \%)$ había fracasado el tratamiento previo con minoxidil tópico al $5 \%$ dos veces por día como monoterapia 0 con finasteride oral durante al menos 1,5 a 2 años.

Se evaluó la eficacia mediante fotografía clínica y tricoscopía digital a las 24 semanas. En $14(43,8 \%)$ pacientes, se observó una mejoría de diferente magnitud cuando se utilizó la fotografía clínica como medio de evaluación, mientras que en $25(78,1 \%)$ pacientes se observó mejoría en la tricoscopía digital. La zona de mayor respuesta con la utilización de ambos métodos fue el vértice.

El minoxidil oral, en dosis de 1,25 mg/día, puede utilizarse para el tratamiento de la alopecia androgénica. $\mathrm{Si}$ el resultado es subóptimo después de 6 meses de tratamiento, se pueden usar dosis más altas (2,5-5 mg/día). Jha AK, Sonthalia S, Zeeshan MD, Vinay K. Efficacy and safety ofvery-low-dose oral minoxidil $1.25 \mathrm{mg}$ in male androgenetic alopecia. J Am Acad Dermato/ 2020;83:1491-1493.

TRATAMIENTO DEL MELASMA. REVISIÓN BASADA EN LAEVIDENCIA

El melasma es una alteración pigmentaria crónica y recurrente, muchas veces refractaria a los tratamientos. Afecta principalmente a mujeres en edad fértil y tiene un impacto negativo en la calidad de vida.

Los tratamientos tanto tópicos como sistémicos son múltiples y la respuesta a ellos es variada.

Se comparó la eficacia de diferentes tratamientos: hidroquinona sola; combinación de una crema triple con tretinoína, hidroquinona y esteroides tópicos; exfoliaciones químicas con diferentes ácidos; terapia con láser; dispositivos que utilizan luz, como la luz pulsada intensa, entre otros.

Elácido tranexámico por vía oral es un tratamiento con resultados prometedores para el melasma recurrente moderado a severo.

La hidroquinona tópica es la más estudiada, tiene un bajo riesgo de efectos adversos y muy buena respuesta terapéutica. La crema triple con hidroquinona, tretinoína y un corticosteroide tópico fue el tratamiento más eficaz. El uso de protector solar, con un factor mayor 0 igual a 30, es imprescindible como parte del tratamiento contra el melasma.

Las exfoliaciones químicas, así como las terapias con láser y con luz pulsada intensa, tienen resultados variables y mayor riesgo de efectos adversos, sobre todo en los pacientes de fototipo elevado.

Recientemente, se observó que el uso del ácido tranexámico por vía oral es eficaz en el tratamiento del melasma moderado a severo o recalcitrante, pero se requieren más estudios para evaluar su eficacia y seguridad a largo plazo. Mckesey J, Tovar-Garza A, Pandya AG. Melasma treatment: An evidence-based review. Am J Clin Dermatol 2020;21:173-225. 